\title{
Steady-state quantum interference in resonance fluorescence
}

\author{
D A Cardimona, M G Raymer and C R Stroud Jr \\ The Institute of Optics, University of Rochester, Rochester, NY 14627, USA
}

Received 15 June 1981, in final form 27 August 1981

\begin{abstract}
It is shown that when a monochromatic laser couples a single atomic ground level to two closely spaced excited levels the system can be driven into a state in which quantum interference prevents any fluorescence from the excited levels, regardless of the intensity of the exciting field. This steady-state interference occurs only at a particular excitation frequency which depends on the separation of the excited states and the relative size of the two transition dipole matrix elements. The results are derived from the density matrix equations of motion. It is shown that a correct description of the effect requires the inclusion of generalised Einstein $A$ coefficients which are usually neglected in phenomenological damping theories. A dressed-state analysis is introduced to simplify the generalisation to atoms having more complex manifolds of excited states. Analogous interferences in multiphoton absorption and ionisation are also discussed briefly.
\end{abstract}

\section{Introduction}

When a laser resonantly excites a many-level atomic system there are a number of quantum interference effects which can occur in the absorption and subsequent fluorescence. The possibility of transient quantum beats in the spontaneous emission following impulse excitation was first proposed by Breit (1933), and more recently discussed by Franken (1961), Chow et al (1975), Herman et al (1975) and Khoo and Eberly (1978). Quantum beats were first observed in a double-resonance experiment by Dodd et al (1959).

In this paper we show that the possibility also exists for a 'steady-state quantum beat', i.e. an exact vanishing of the spontaneous emission from a multi-level atom continuously excited by a monochromatic laser field. This may occur if the system satisfies three conditions. First, the incident laser field must be tuned to a particular frequency. Second, the excited states must have exactly the same angular momentum quantum numbers and differ only in the principal quantum number. Finally, the decay must be to a single ground state. This is a stringent, but achievable, set of requirements.

Normally, quantum beats occur in only certain directions and certain polarisations rather than in the total fluorescence. This was pointed out by Breit (1933), Macek (1969), Stenholm (1977) and Knight (1980), and is true here also, if the second condition given above is violated. But, if all three conditions are satisfied, the total fluorescence vanishes.

Steady-state quantum interference may be understood intuitively in terms of a charge cloud model. In the case of two upper states, the exciting laser field will generate a coherent oscillating dipole moment for each transition. The dipoles will each oscillate 
at the driving field frequency with a phase relation which depends on the detuning of the laser from each resonance. At a particular detuning the two dipoles will be equal in amplitude and exactly opposite in phase. The total dipole moment will then vanish if the polarisations of the two dipoles are identical. In that case the atom will be decoupled from the radiation field and there will be no fluorescence. This is the case only when all three of the above conditions are met.

A proper theory of the spontaneously emitted fluorescence requires a quantum electrodynamic treatment. Milonni (1976) and Renaud (1976) have carried out such a quantum treatment for multi-level systems. They found that in addition to the usual Einstein $A$ coefficients there are generalised damping coefficients which couple two transitions having a common lower level. These damping coefficients were originally introduced by Landau (1927) in his classic paper in which the density matrix approach to quantum mechanics was first formulated. These terms are not generally included in phenomenological semiclassical damping theories, but must be included in our treatment because the coherence between the two upper-state amplitudes affects the spontaneous damping rate.

We include these generalised damping terms in a set of density matrix equations of motion, and derive the predicted steady-state absorption rate as a function of the exciting field frequency. This absorption spectrum has a zero at the field frequency at which the two dipole moments cancel.

We extend the theory to include the case of an arbitrary number of excited levels. That calculation is more conveniently carried out using dressed-state rate equations similar to those developed by Cohen-Tannoudji and Reynaud (1977) and by Whitley and Stroud (1976). It is found that there may be up to $N-1$ zeros in the absorption spectrum when there are $N$ excited states all coupled to a common lower level.

Knight $(1979,1980)$ and Coleman and Knight (1981) have shown that analogous generalised damping coefficients are important in photoionisation. We will discuss the connection between the interference in the present problem and that in two-channel photoionisation.

\section{Absorption line shape}

Consider a model atom, as shown in figure 1, having two upper states $|2\rangle$ and $|3\rangle$ which are coupled by dipole matrix elements to a single common ground state $|1\rangle$. A laser field

$$
E(t)=E_{0}\left(\varepsilon \mathrm{e}^{-\mathrm{i} \omega t}+\mathrm{CC}\right)
$$

is tuned to the vicinity of the resonance frequencies $\omega_{21}$ and $\omega_{31}$. In the frame rotating with the applied field, the density matrix equations are (Milonni 1976, Renaud 1976)

$$
\begin{gathered}
\dot{r}_{33}=-2 \gamma_{3} r_{33}-\frac{1}{2} \mathrm{i}\left(\Omega_{13} r_{13}-\mathrm{CC}\right)-\left\{\left(\gamma_{3112}-\mathrm{i} S_{3112}\right) r_{32}+\mathrm{CC}\right\} \\
\dot{r}_{22}=-2 \gamma_{2} r_{22}-\frac{1}{2} \mathrm{i}\left(\Omega_{12} r_{12}-\mathrm{CC}\right)-\left\{\left(\gamma_{2113}-\mathrm{i} S_{2113}\right) r_{23}+\mathrm{CC}\right\} \\
\dot{r}_{31}=-\left[\mathrm{i}\left(\Delta-\omega_{32}-S_{3}\right)+\gamma_{3}\right] r_{31}+\frac{1}{2} \mathrm{i} \Omega_{13}\left(r_{33}-r_{11}\right)+\frac{1}{2} \mathrm{i} \Omega_{12} r_{32}-\left\{\left(\gamma_{3112}^{*}+\mathrm{i} S_{3112}^{*}\right) r_{21}\right\} \\
\dot{r}_{21}=-\left[\mathrm{i}\left(\Delta-S_{2}\right)+\gamma_{2}\right] r_{21}+\frac{1}{2} \mathrm{i} \Omega_{12}\left(r_{22}-r_{11}\right)+\frac{1}{2} \mathrm{i} \Omega_{13} r_{23}-\left\{\left(\gamma_{2113}^{*}+\mathrm{i} S_{2113}^{*}\right) r_{31}\right\} \\
\dot{r}_{32}=\left[\mathrm{i}\left(\omega_{32}+S_{3}-S_{2}\right)-\gamma_{2}-\gamma_{3}\right] r_{32}+\frac{1}{2} \mathrm{i} \Omega_{12}^{*} r_{31}-\frac{1}{2} \Omega_{13} r_{12} \\
\quad-\left\{\left(\gamma_{2113}-\mathrm{i} S_{2113}\right) r_{33}+\left(\gamma_{3112}^{*}+\mathrm{i} S_{3112}^{*}\right) r_{22}+\mathrm{i}\left(S_{1321}-S_{1231}^{*}\right) r_{11}\right\}
\end{gathered}
$$




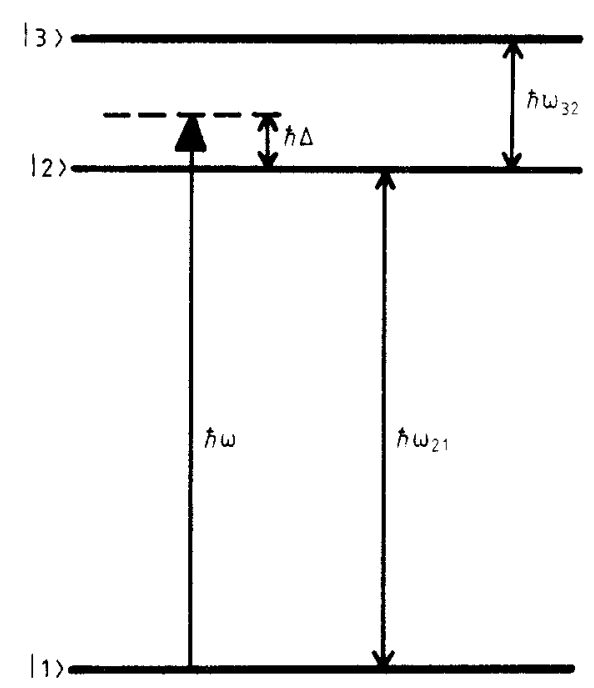

Figure 1. A three-level atom, with two upper states connected by dipole transitions to the ground state, is excited by a continuous laser with frequency $\omega$.

where $\Omega_{i j}=2 \boldsymbol{d}_{i j} \cdot \boldsymbol{\varepsilon}^{*} E_{0} / \hbar$ is the Rabi frequency and $\boldsymbol{d}_{i j}=\langle i|\hat{\boldsymbol{d}}| j\rangle$ is the dipole matrix element for the $i-j$ transition, $\varepsilon$ is the unit polarisation vector of the laser field, and $\Delta=\omega-\omega_{21}$ is the detuning of the laser from atomic level $|2\rangle$. The rotating wave approximation has been used. The spontaneous damping and frequency shift terms are

$$
\begin{aligned}
\gamma_{a b c d} & \equiv\left(\frac{2}{3 \hbar c^{3}}\right) \boldsymbol{d}_{a b} \cdot \boldsymbol{d}_{c d}\left|\omega_{c d}\right|^{3} \\
S_{a b c d} & \equiv\left(\frac{2}{3 \pi \hbar c^{3}}\right) \boldsymbol{d}_{a b} \cdot \boldsymbol{d}_{c d} \mathbf{P} \int \frac{\omega^{3} \mathrm{~d} \omega}{\omega+\omega_{c d}}
\end{aligned}
$$

and we have used the abbreviations $\gamma_{j}=\gamma_{1 j i 1}$ and $S_{j}=S_{1331}+S_{1221}-S_{i 11 j}$ for $j=2$ and 3. Population is assumed to be conserved, so that

$$
r_{11}+r_{22}+r_{33}=1 \text {. }
$$

Note that $\gamma_{2}$ and $\gamma_{3}$ are the usual Einstein $A$ coefficients for spontaneous damping, while $S_{2}$ and $S_{3}$ are the usual Lamb shifts of levels $|2\rangle$ and $|3\rangle$ before mass renormalisation. The other terms involving $\gamma$ and $S$, and enclosed in curly brackets \{\} in equation (1), account for the effect of the coherence between the 1-2 and 1-3 transitions on the damping and shifting. These generalised terms have normally been left out of previous treatments of multi-level atoms interacting with radiation, although their existence is generally known. If the separation $\omega_{32}$ between the upper levels is large compared to the shifts of the lines, the shifts may be considered to be incorporated in the atomic frequencies $\omega_{21}$ and $\omega_{31}$, and thus they will be omitted in equation (1). This point will be discussed in detail in a subsequent paper.

The rate of absorption of photons from the laser field is

$$
I(\Delta)=\frac{1}{2} \mathrm{i}\left(\Omega_{12}^{*} r_{21}+\Omega_{13}^{*} r_{31}-\mathrm{CC}\right) .
$$

This will be evaluated analytically in the case that the dipole moments $d_{12}$ and $d_{13}$ are parallel, i.e. $\boldsymbol{d}_{13}=z \boldsymbol{d}_{12}$, where $z$ is real. No further loss of generality results if $\Omega_{12}$ and 
$\Omega_{13}$ are chosen to be real. Then the steady-state absorption line shape is found from equations (4) and (1) (after lengthy algebra) to be

$$
I(\Delta)=\frac{2 \gamma_{2} \Omega_{12}^{2}\left[\Delta\left(z^{2}+1\right)-\omega_{32}\right]^{2}}{D(\Delta)}
$$

where

$D(\Delta)=4 \Delta^{2}\left(\Delta-\omega_{32}\right)^{2}+2\left(\Omega_{12}^{2}+2 \gamma_{2}^{2}\right)\left[\Delta\left(z^{2}+1\right)-\omega_{32}\right]^{2}+4 z^{2} \Omega_{12}^{2} \Delta \omega_{32}+\frac{1}{4}\left(z^{2}+1\right) \Omega_{12}^{4}$.

To obtain this result we have used $\Omega_{13}=z \Omega_{12}$ and the approximation $\omega_{32} \ll \omega_{21}, \omega_{31}$.

Equation $(5 a)$ indicates that when the laser is detuned from level $|2\rangle$ by an amount equal to

$$
\Delta=\omega_{32} /\left(z^{2}+1\right)
$$

the steady-state absorption rate becomes identically zero. In the special case that $\boldsymbol{d}_{12}=\boldsymbol{d}_{13}$, this occurs when $\Delta=\frac{1}{2} \omega_{32}$, that is, when the laser is tuned midway between levels $|2\rangle$ and $|3\rangle$.

The exact vanishing of the absorption rate (and thus the total fluorescence rate) is the main point of this paper, and may be understood intuitively as a cancellation of the amplitudes of the oscillating dipoles of the two transitions. The cancellation of the dipole amplitudes $r_{21}$ and $r_{31}$ arises solely through the off-diagonal damping terms $\left(\gamma_{3112}\right.$, etc) in equation (1) and thus we see that if $\boldsymbol{d}_{12} \cdot \boldsymbol{d}_{13}=0$ (the dipoles are orthogonal), no such cancellation occurs. It is notable that the cancellation persists even at high laser intensities.

In order to study these results in more detail, we have plotted in figure 2 the absorption rate $I(\Delta)$ as a function of the laser detuning $\Delta$, for the case in which $\left|\boldsymbol{d}_{12}\right|=\left|\boldsymbol{d}_{13}\right|$ and the laser is polarised along the $x$ axis $(\varepsilon=x)$, with a field amplitude that is varied to give three different values for the Rabi frequency $\Omega_{12}$. Figure $2(a)$ shows the absorption profile obtained when $d_{12}$ and $\boldsymbol{d}_{13}$ are both parallel to the $x$ axis, as given in equation (5). Note that at high laser intensity the quantum interference effect prevents the two peaks from power broadening into one another. Rather, a zero is seen in the absorption profile at $\Delta=\frac{1}{2} \omega_{32}$. Figure $2(b)$ shows the absorption profile obtained $\dagger$ when $\boldsymbol{d}_{12}=d(\boldsymbol{x}-\mathrm{i} \boldsymbol{y})$ and $\boldsymbol{d}_{13}=d(\boldsymbol{x}+\mathrm{i} \boldsymbol{y})$. There is no interference minimum in this profile because two orthogonal polarisations cannot produce steady-state interference.

For parallel polarisations, even in the case that the two upper levels have natural widths comparable to or larger than their separation, equation (5) predicts a zero in the absorption profile. This effect allows sub-natural resolution of the atomic lines. A related discussion has been given in the special case of weak incident fields by Morozov and Shorygin (1964).

\section{Multi-level atoms and the dressed-state approach}

The quantum interference effect discussed above for the hypothetical three-level atom is also found to occur in an atom with many excited levels. For example, an atom with a ${ }^{1} \mathrm{~S}_{0}$ ground state may be excited by a linearly polarised laser field to produce a coherent superposition of a large number of closely spaced ${ }^{1} \mathrm{P}_{1}\left(m_{j}=0\right)$ Rydberg states. We will

\footnotetext{
$†$ The analytical expression for $I(\Delta)$ is quite lengthy in this case, and thus is not reproduced here.
} 

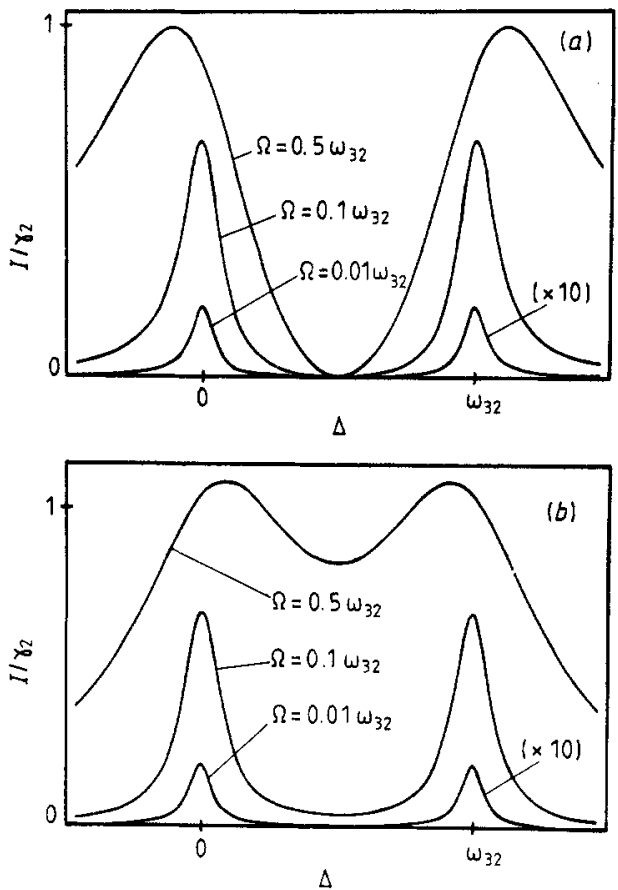

Figure 2. The absorption rate, $I(\Delta)$, divided by the natural line width, $\gamma_{2}$, plotted against the laser detuning, $\Delta$, for the three-level atom of figure 1 . The transition dipole moments are $(a)$ parallel $\left(\boldsymbol{d}_{12}=\boldsymbol{d}_{13}=\boldsymbol{d} \boldsymbol{x}\right)$ or $(b)$ orthogonal $\left(\boldsymbol{d}_{12}=d(\boldsymbol{x}-\mathrm{i} \boldsymbol{y})=\boldsymbol{d}_{13}^{*}\right)$. In each case, the applied field amplitude, $\boldsymbol{E}=2 E_{0} \boldsymbol{x}$, is varied to give three values for the Rabi frequency $\Omega=2 d E_{0} / \hbar$. The natural line widths were taken to be $\gamma_{2}=\gamma_{3}=0.05 \omega_{32}$.

see below that in this case the laser absorption goes exactly to zero at a particular detuning between every pair of Rydberg states. In principle, one may solve numerically the appropriate many-level density matrix equations analogous to equation (1), but this approach rapidly becomes cumbersome as the number of levels is increased. We have found it more efficient to use the method of dressed states.

The dressed states are the eigenvectors of the combined atom-field system. With the laser field represented by a Fock state $|n\rangle$, where $n$ is the number of photons, the product states $|n\rangle|j\rangle$, where $j$ labels the atomic state, form the basis set for the problem. The dressed states, denoted as $|n, \mu\rangle$, satisfy

$$
H|n, \mu\rangle=\mathscr{E}_{n \mu}|n, \mu\rangle
$$

where $H$ is the total (time-independent) Hamiltonian, including atomic and field energies and the dipole interaction energy $-\hat{d} \cdot \hat{\boldsymbol{E}}$.

Consider an atom with $N$ upper levels coupled to a common ground level $|g\rangle$. If this atom is excited by a near-resonant laser field of frequency $\omega$, the Hamiltonian in the rotating wave approximation is

$$
H=\left(n \hbar \omega+\hbar \omega_{g}\right) I-\frac{1}{2} \hbar\left(\begin{array}{ccccl}
0 & -i \Omega_{g 1} & -i \Omega_{g 2} & \cdots & -i \Omega_{g N} \\
i \Omega_{g 1}^{*} & 2 \Delta & 0 & \cdots & 0 \\
i \Omega_{g 2}^{*} & 0 & 2\left(\Delta-\omega_{21}\right) & \cdots & 0 \\
\vdots & \vdots & 0 & & \vdots \\
i \Omega_{g N}^{*} & 0 & \cdots & & 2\left(\Delta-\omega_{N 1}\right)
\end{array}\right)
$$


where $I$ is the identity matrix, $\hbar \omega_{\mathrm{g}}$ is the ground-state energy, and $\Delta=\omega-\omega_{1 \mathrm{~g}}$. This Hamiltonian may be diagonalised to find the eigenvalues $\mathscr{E}_{n \mu}$ and the dressed states, which we write as

$$
|n, \mu\rangle=c_{g \mu}|n\rangle|g\rangle+\sum_{j=1}^{N} c_{j \mu}|n-1\rangle|j\rangle \quad \mu=1, \ldots, N+1 .
$$

When the energy separations of the dressed states exceed their line widths, all coherent effects will be included in the dressed states themselves, leaving only the incoherent process of spontaneous emission to change the level populations. This formalism is accurate to second order in the ratio of the line widths to the separations between the dressed levels. The dressed-atom dynamics are governed by the simple rate equation

$$
\dot{p}_{\mu}=\sum_{\nu \neq \mu}^{N+1}\left(\gamma_{\nu \mu} p_{\nu}-\gamma_{\mu \nu} p_{\mu}\right)
$$

where $p_{\mu}$ is the sum over $n$ of the populations of the dressed states $|n, \mu\rangle$, and the spontaneous transition rates are given by Fermi's golden rule as

$$
\gamma_{\mu \nu}=\frac{4 \omega_{\mu \nu}^{3}\left|d_{\nu \mu}\right|^{2}}{3 \hbar c^{3}}
$$

where

$$
\begin{aligned}
\boldsymbol{d}_{\nu \mu} & =\langle n-1, \nu|\hat{\boldsymbol{d}}| n, \mu\rangle \\
& =c_{\mathrm{g} \nu}^{*} \sum_{j=1}^{N} c_{j \mu} \boldsymbol{d}_{g j}
\end{aligned}
$$

and

$$
\omega_{\mu \nu}=\hbar^{-1}\left(\mathscr{C}_{n \mu}-\mathscr{C}_{n-1, \nu}\right)
$$

Note that if there exists a dressed state with amplitudes $c_{j \mu}$ such that $\boldsymbol{d}_{\nu \mu}$ is zero for some $\mu$ and all $\nu$, then the rates $\gamma_{\mu \nu}$ out of that state will all be zero. Population will flow into that state and be unable to leave. So, in the steady state, no transitions will occur.

The rate $I(\Delta)$ of energy absorption from the laser field is equal to the rate of atomic fluorescence in the steady state. This rate is found by summing the integrated intensities $I_{\mu \nu}$ of the individual resonance fluorescence spectral components

$$
\begin{aligned}
& I(\Delta)=\sum_{\nu=1}^{N+1} \sum_{\mu=1}^{N+1} I_{\mu \nu} \\
& I_{\mu \nu}=\hbar \omega_{\mu \nu} \gamma_{\mu \nu} p_{\mu} .
\end{aligned}
$$

The populations $p_{\mu}$ are determined by the steady-state solution of the rate equations (10). Carrying out this procedure numerically for the three-level atom of figure 1 , we find that the absorption profile $I(\Delta)$ agrees with that shown in figure 2 . The quantum interference minimum in figure 2(a) may be interpreted as being due to the presence of a particular coherent superposition of atomic states (a dressed state) which is stable with respect to spontaneous decay.

An interesting point is that in the dressed-state approach, no explicit mention need be made of the generalised damping terms which appear in the density matrix equations 
(1). The coherence-dependent damping is automatically accounted for in the dressedstate approach. Also, the dressed-state rate equations exhibit a computational advantage over the density matrix equations in extensions to multi-level atoms. Results for an $N$-level atom require the solution of only $N$ simultaneous equations in the dressed-state approach, rather than the $N^{2}$ equations of the density matrix approach.

To illustrate results obtained using the multi-level dressed-state approach, we considered a seven-level atom with six equally spaced upper states all connected by dipole transitions to the ground state. The eigenvectors of the $7 \times 7$ Hamiltonian matrix were obtained numerically, allowing the calculation of the forty nine spontaneous damping rates $\gamma_{\mu \nu}$. The seven dressed-state rate equations were then solved numerically in the steady state, and the total fluorescence (absorption) rate $I(\Delta)$ was calculated for different detunings $\Delta$. Figures 3 and 4 show the resulting absorption profiles obtained with three different values of the applied field amplitude. Figure 3 shows the case in which all six dipole matrix elements $\boldsymbol{d}_{g j}$ are parallel and equal in magnitude. A zero is found in the profile between each pair of upper levels, even at high fields. Figure 4 shows the case in which the first, third, and fifth dipole matrix elements are of the same polarisation, while the other three are orthogonal to these. In this case no interference structure is found at high fields.
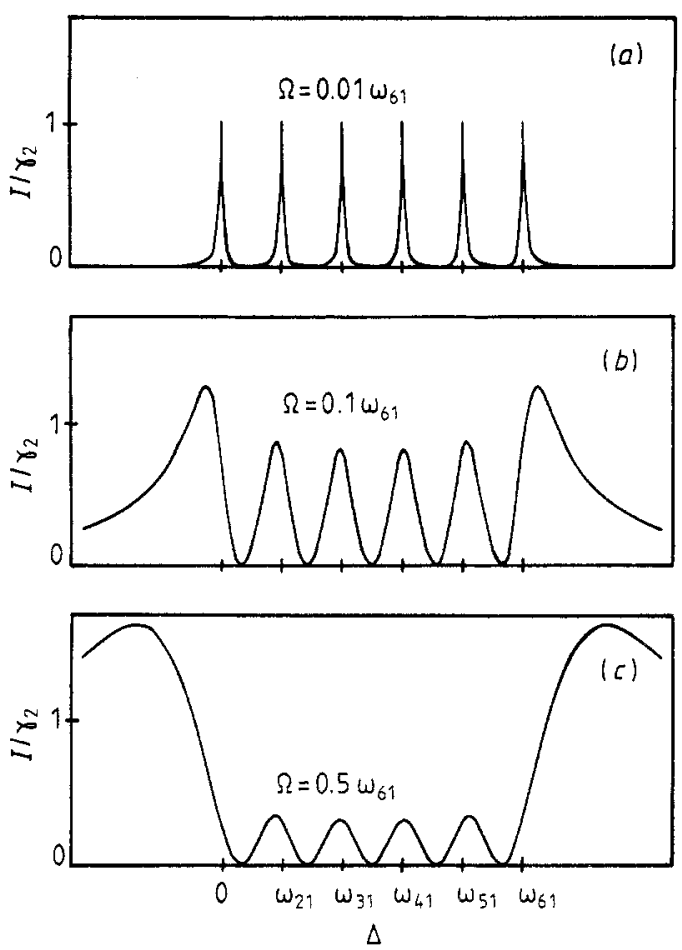

Figure 3. The absorption rate, $I(\Delta)$, divided by the natural line width $\gamma_{2}$, plotted against laser detuning, $\Delta$, for a seven-level atom having six equally spaced upper states all connected by dipole transitions to the ground state $|g\rangle$. All transition dipole moments are equal in magnitude and parallel $\left(d_{g 1}=d_{g 2}=\ldots=d_{g 6}=d x\right)$. The applied field amplitude, $E=2 E_{0} x$, is varied in $(a)-(c)$ to give three values for the Rabi frequency $\Omega=2 d E_{0} / \hbar$. The natural line widths are assumed to be much less than $\omega_{61}$. 

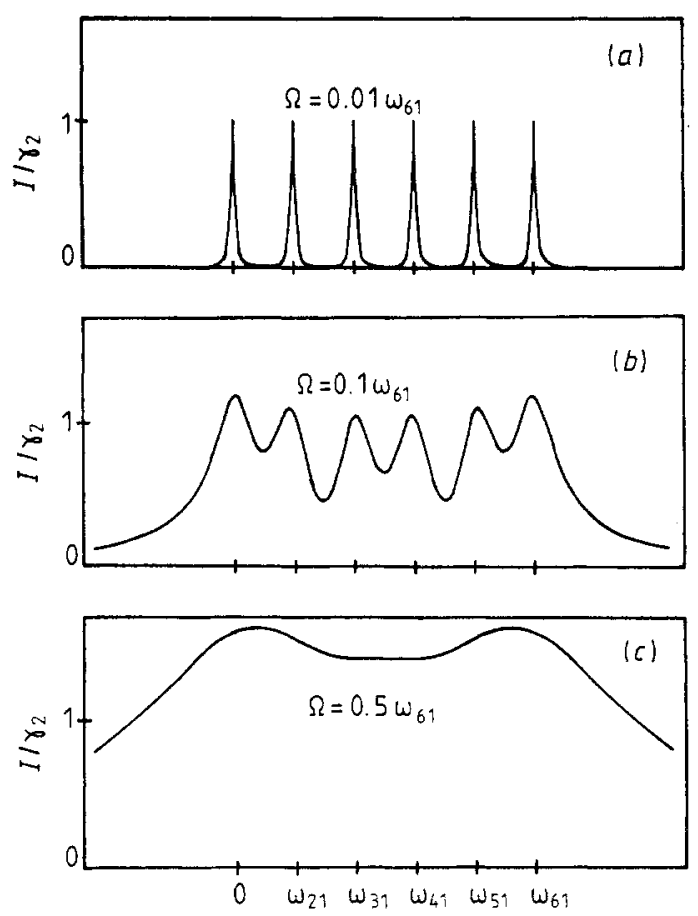

$\Delta$

Figure 4. The same as figure 3 , except that adjacent transition dipole moments are orthogonal, such that $d_{g 1}=d_{83}=d_{g 5}=d(x-\mathrm{i} y)$ and $d_{g 2}=d_{g 4}=d_{86}=d(x+\mathrm{i} y)$.

\section{Extension to other cases}

We have found that steady-state quantum interference may cause complete cancellation in the total resonance fluorescence because we have restricted our attention to atoms having a single (non-degenerate) ground state. If we expand our treatment to include atoms having multiple ground states, the generalised damping terms in the density matrix equations are obtained by summing over all ground states, including degenerate states. Breit (1933), Macek (1969), Stenholm (1977) and Knight (1980) proved that these sums are zero if either the total angular momentum quantum number $J$ or the magnetic quantum number $m_{J}$ are different for the two excited states. In this case, neither transient quantum beats nor the steady-state quantum beat described here will occur in the total fluorescence, but only in certain polarisations. On the other hand, if the excited states are Rydberg states with different principal quantum numbers, it is possible for their $J$ and $m_{J}$ values to be equal, thereby allowing quantum interference to affect the total fluorescence. Even in this case, the interference will not go exactly to zero if there are multiple ground states. We can, however, create conditions in which only a single ground state participates by optical pumping (Abate 1974).

The processes of resonance fluorescence, Raman scattering, two-photon absorption, and two-photon ionisation all have very similar features, as can be seen in figure 5 , so the steady-state quantum interference effect discussed above should have an analogue in each of these cases. To get a zero in the total steady-state resonance fluorescence, we found above that the net excited dipole moment to the ground state, 


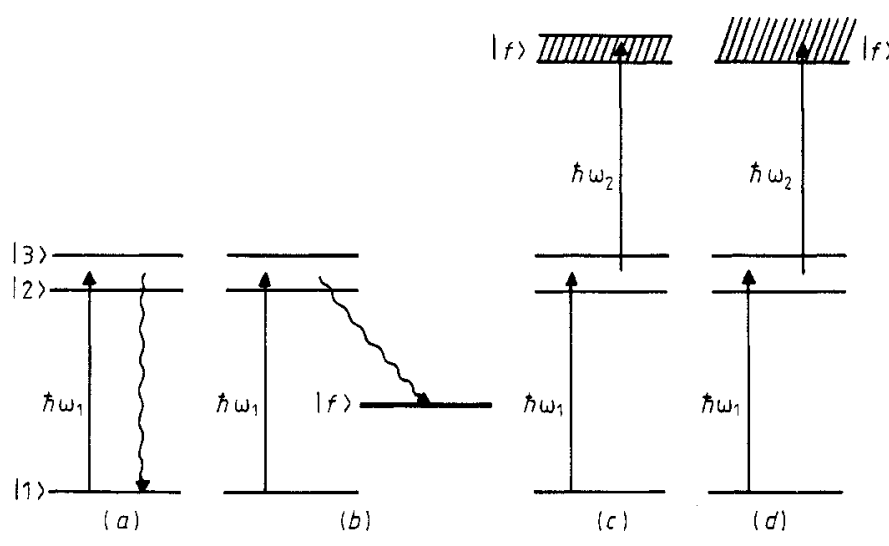

Figure 5. Four processes in which the possibility exists for steady-state quantum interference involving two intermediate states: $(a)$, resonance fluorescence as studied here; $(b)$, Raman scattering; $(c)$, two-photon absorption and $(d)$ two-photon ionisation.

$d_{1}=a d_{12}+b d_{13}$ (where $a$ and $b$ are functions of detuning and laser intensity), must be equal to zero so that the dipole transition rate to the continuum of vacuum states which is proportional to $\left|d_{1} \cdot \varepsilon_{s}\right|^{2}$ will be zero regardless of the polarisation $\boldsymbol{\varepsilon}_{\mathrm{s}}$ of the fluorescent emission. The condition for Raman scattering to vanish in the steady state is almost identical. We must have the net dipole moment to the final state $|f\rangle, \boldsymbol{d}_{f}=a \boldsymbol{d}_{f 2}+b \boldsymbol{d}_{f 3}$, be zero, so that $\left|\boldsymbol{d}_{f} \cdot \boldsymbol{\varepsilon}_{\mathbf{s}}\right|^{2}$ is zero for all polarisations $\boldsymbol{\varepsilon}_{\mathbf{s}}$. Note that if the ratio of $\boldsymbol{d}_{f 2}$ to $\boldsymbol{d}_{f 3}$ is the same as the ratio of $\boldsymbol{d}_{12}$ to $\boldsymbol{d}_{13}$, then the Raman scattering and the resonance fluorescence will vanish at the same laser frequency. The two-photon absorption and ionisation cases have somewhat less restrictive conditions that must be satisfied for zero steady-state population in the upper states $|f\rangle$ because the experimenter controls the polarisation of the second field at $\omega_{2}$. Now $\varepsilon_{\mathrm{s}}$ is equal to the polarisation vector of the laser at frequency $\omega_{2}$, and the condition $\boldsymbol{d}_{f} \cdot \varepsilon_{\mathrm{s}}=0$ just means that we must have the net Rabi frequency $a \Omega_{f 2}+b \Omega_{f 3}$ vanish. This relaxes the restriction that the dipole moments $d_{f 2}$ and $d_{f 3}$ be parallel. Thus, it should be much easier to produce the steady-state quantum beat effect in two-photon absorption and ionisation. In fact, the deep interference minimum in the two-photon ionisation cross section of caesium atoms recently observed by Morellec et al (1980) and originally predicted by Bebb (1966) is a manifestation of this steady-state quantum beat.

The conditions for seeing the steady-state beat in spontaneous emission are more restrictive than they are in two-photon photoionisation, two-photon absorption etc, because spontaneous emission is normally a spherically symmetric process. In the other processes a second field breaks the symmetry and restricts the potential final states, making complete interference more easily attainable.

\section{Discussion and conclusions}

We have shown that we can completely eliminate the steady-state absorption, and therefore also the steady-state resonance fluorescence, of a three-level atom having two closely spaced excited states, each coupled to a common ground state by parallel dipole moments, simply by tuning the incident laser field appropriately. We introduced a 
dressed-state rate equation approach for the generalisation to atoms having many upper levels, because it offered the advantage of having fewer simultaneous equations to be solved than found in the density matrix approach. We found that when the upper-level separation is much greater than the natural line widths, the dressed-state transition rates automatically contain within them the normally ignored generalised damping terms that must be explicitly included in the density matrix equations. As for the case of two upper levels, we found that the dipole matrix elements connecting any number of upper states to a common ground state must be parallel for quantum interference to affect the total steady-state resonance fluorescene. Rather surprisingly, if all of the dipole moments are parallel, the scattered intensity goes to zero as the incident field is tuned between each pair of excited states.

The interference effect is dramatic when the excited states overlap appreciably, as they do when there is power broadening. The zeros are found to persist even when the levels would otherwise be completely mixed by an intense laser field.

\section{Acknowledgments}

We would like to thank P Knight, P Milonni and W Molander for helpful discussions. We would also like to acknowledge the support of the US Army Research Office under contract DAAG29-81-K-0134, and Bausch and Lomb. The algebra leading to figure $4(b)$ was performed using MACSYMA, the algebraic manipulation system developed by the Mathlab group of the MIT Laboratory for Computer Science. P.Drummond and J Eberly provided much appreciated help in using MACSYMA.

\section{References}

Abate J A 1974 Opt. Commun. 10 269--72

Bebb H B 1966 Phys. Rev. 149 25-32

Breit G 1933 Rev. Mod. Phys. 5 91-140

Chow W W, Scully M O and Stoner J O Jr 1975 Phys. Rev. A 11 1380-8

Cohen-Tannoudji C and Reynaud S 1977 J. Phys. B: At. Mol. Phys. 10 345-63

Coleman P E and Knight P L 1981 Phys. Lett. 81A 379-82

Dodd J N, Fox W N, Series G W and Taylor M J 1959 Proc. Phys. Soc. 74 789-90

Franken P A 1961 Phys. Rev. 121 508-12

Herman R M, Grotch H, Kornblith R and Eberly J H 1975 Phys. Rev. A 11 1389-96

Khoo I C and Eberly J H 1978 Phys. Rev. A 18 2184-91

Knight P L 1979 Opt. Commun. 31 148-52

1980 Opt. Commun. 32 261-5

Landau L 1927 Z. Phys. 45430 (Engl. trans. 1967 Collected Papers of L D Landau, ed D Ter Haar (New York: Gordon and Breach) pp 8-18)

Macek J 1969 Phys. Rev. Lett. 23 1-2

Milonni P W 1976 Phys. Rep. C 25 1-81

Morellec J, Normand D, Mainfray G and Manus C 1980 Phys. Rev. Lett, 44 1394-7

Morozov V A and Shorygin P P 1966 Opt. Spektrosk. 20 115-20

Renaud B 1976 PhD Thesis University of Rochester, unpublished

Stenholm S 1977 Frontiers in Laser spectroscopy vol 1, ed R Balian, S Haroche and S Liberman (Amsterdam: North-Holland)

Whitley R M and Stroud C R Jr 1976 Phys. Rev. A 14 1498-513 